\title{
An X-ray template assessment for distal radial fractures
}

\author{
P. V. van Eerten · R. Lindeboom • A. E. Oosterkamp • \\ J. C. Goslings
}

Received: 16 March 2007 / Published online: 11 July 2007

(C) Springer-Verlag 2007

\begin{abstract}
Background The current method for radiological measurements on plain X-rays of distal radius fractures is unreliable. We examined the reproducibility of a new X-ray assessment technique-where the uninjured side is used as a template for the injured side-compared to the conventional assessment technique.

Methods X-rays of 30 patients with a unilateral distal radial fracture were included reflecting the prevalence of AO fracture types in clinical practice. Eight experienced observers assessed these X-rays on two separated occasions (2-month interval) using the traditional measurement technique and the template technique. Reproducibility of the Xray assessments was quantified by intraclass correlations and weighted kappa coefficients.

Results The reproducibility of the radial length measurement did not improve nor did the volar angulation measurement. However, marked improvement in reproducibility was observed for the radial inclination measurement, the kappa increased from 0.36 (95\% CI; 0.30-0.41) to 0.49 (95 $\% \mathrm{CI} ; 0.43-0.55)$ in the template technique. As a result, the classification of the reduction results (Lidström score)
\end{abstract}

P. V. van Eerten $(\bowtie) \cdot$ A. E. Oosterkamp

Department of Surgery/Traumatology,

Máxima Medical Center, PO Box 90052 ,

5600 PD Eindhoven, The Netherlands

e-mail: p.vaneerten@mmc.nl

R. Lindeboom

Department of Clinical Epidemiology en Biostatistics,

Academic Medical Center, Amsterdam, The Netherlands

J. C. Goslings

Trauma Unit Department of Surgery,

Academic Medical Center, Amsterdam, The Netherlands greatly improved. The overall kappa for the Lidström score improved from 0.37 (95\% CI; 0.31/0.43) to 0.59 $(0.52 / 0.63)$.

Conclusion The assessment technique using the uninjured side as a template for the injured side resulted only in an improved reproducibility of the radial inclination measurement which in turn resulted in an improved classification reproducibility of the reduction results.

Keywords Radius $\cdot$ Radius fracture $\cdot \mathrm{X}$-ray .

Classification $\cdot$ Distal radial fractures

\section{Introduction}

The important factors for decision-making in the treatment of distal radial fractures include the patient's vitality, fracture classification, fracture dislocation and soft tissue condition. Distal radial fracture deformities are quantified by X-ray measurements which are also used to classify the result after reduction (e.g., good or fair result) [1, 2]. However, the clinical usefulness of X-ray measurements is hampered by their limited reproducibility [3, 4]. It is also recognized that the modest reproducibility of X-ray deformations is one of the keystones for the lack of reproducibility in the treatment of distal radial fractures [3-5]. Clearly, when measurements cannot be made reliably, a straightforward clinical comparison of treatment techniques or reduction results is impossible, making it difficult to come to level 1 evidence-based treatment results and advices.

Other studies that have evaluated sources of variance in the assessment of the quantitative X-ray measurements have reported difficulties with respect to the determination of the radial axis in the anterior-posterior (AP) view, which is essential to assess the radial height and the radial inclination. 
This is obviously much less a problem in the lateral view, where the higher reproducibility value was found [6]. A second source of inaccuracy might be the use of the existing normal values for radial length $(11 \mathrm{~mm})$, radial angle $\left(22^{\circ}\right)$ and volar angulation $\left(10^{\circ}\right)$ [7-11], despite the normal variation that exists within the population [12].

To circumvent these difficulties, we designed a technique to assess fracture deformities of the distal radius that is based on the comparison of the injured side to the uninjured side. The approach is based on research findings that indicate that the patients' left and right side are comparable [13]. The aim of the study was to evaluate the reproducibility of the template assessment technique as compared with the direct quantitative measurement technique. We also examined whether the new technique resulted in a more reliable classification of reduction results for distal radius fractures.

\section{Materials and methods}

\section{Inclusion criteria}

Patients aged 18 years or above with a unilateral dislocated distal radial fracture with the history of neither a distal radial fracture nor a distal radial operation were included after informed consent was taken. A stratification for the $\mathrm{AO}$ fracture types was performed to reflect the prevalence in clinical practice at a ratio of 5:2:3 for the AO type A, B and $\mathrm{C}$ distal radial fractures. The standard post-reduction (postero-anterior (PA) and lateral) X-rays and standard distal radius $\mathrm{X}$-rays of the unaffected side of 30 consecutive patients visiting the emergency department were included after stratification for AO fracture type.

Eight experienced observers, 4 trauma surgeons, 3 orthopedic surgeons and 1 trauma radiologist, independently measured the X-rays and subsequently classified the reduction results using the Lidström score (Fig. 1).

In the first direct measurement round, the x-rays were only measured quantitatively. The radial length and the radial inclination were measured on the posterior-anterior plain X-ray film, while the dorsal/volar angulation was measured on the lateral X-ray film. The measurements were performed with a goniometer and a ruler. The normal values for radial length $(11 \mathrm{~mm})$, radial inclination $\left(22^{\circ}\right)$ and volar angulation $\left(10^{\circ}\right)$ were used to assign a Lidström score (Fig. 2).

During the second round, scheduled 2 months later, the template measurement technique of comparing the fractured side with the unaffected side was performed by the same observers measuring the $\mathrm{X}$-rays of the same patients. The observers were instructed to assign a Lidström score using the X-rays of the unaffected distal radius as a

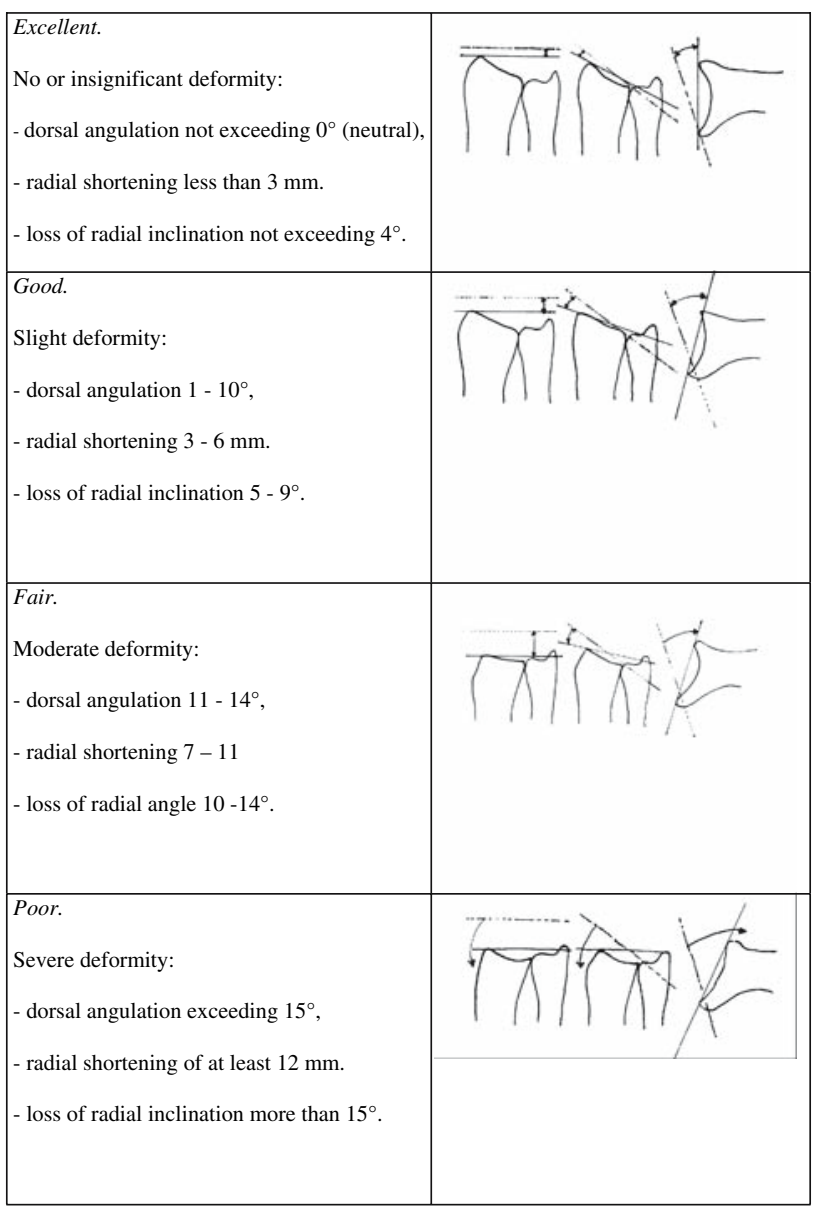

Fig. 1 Anatomical radiological classification for distal radial fractures according to Lidström

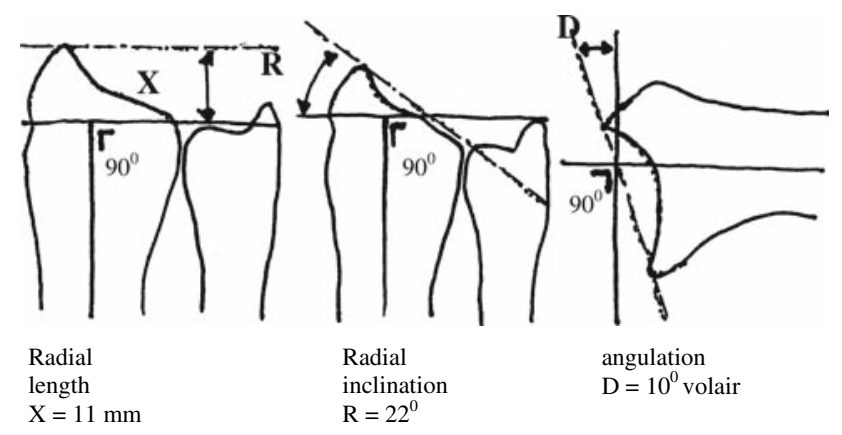

Fig. 2 Normal value measurement of the distal radius

template for the fractured side (Fig. 3). The templates of the unaffected side were made by the investigators on transparent sheets, by tracing the distal radius, ulna and first carpal row from the unaffected side plain PA X-Ray film on a overlaid transparent sheet. For the lateral film, only the distal radius and the lunate bone from the unaffected side were drawn on to the overlaid transparent sheet. The reference points on the AP templates were the ulna and/or the first carpal row (Fig. 3). On the lateral template, the reference 


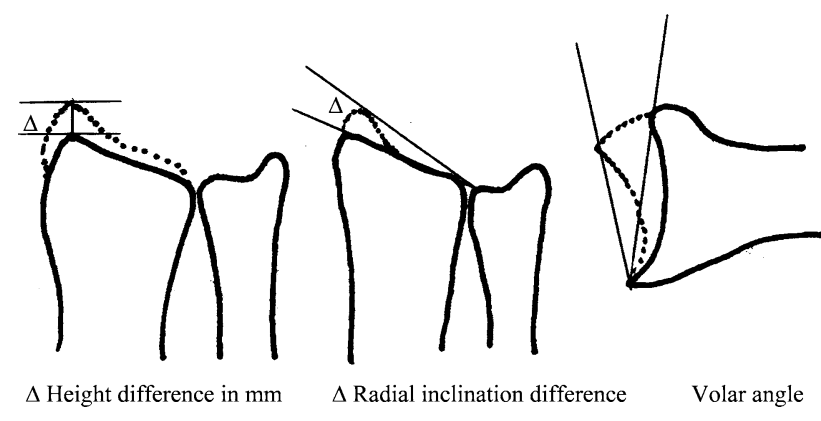

Fig. 3 Measurements of distal radial fractures using the unaffected side as template

points were the radial shaft/and or the lunatum. The measurements again were done with a goniometer and a ruler. And again the results were used to assign a Lidström score.

Statistical analysis

Overall inter-observer reproducibility of quantitative X-ray measurements for the difference in radial length, radial inclination and volar angulation for the eight observers was estimated using intraclass correlation coefficients (case 2,1) [14]. The reproducibility of the ordinal Lidström scoring was quantified using a weighted kappa coefficient (Kw). Since intraclass correlations (ICC) and $\mathrm{Kw}$ are computationally equivalent [15], both were interpreted using the arbitrary classification proposed by Landis and Koch [16]: $<0.00$ poor; $0.00-0.20$ slight; $0.21-0.40$ fair; $0.41-0.60$ moderate; $0.61-0.80$ substantial and $0.81-1.00$ almost perfect agreement. Calculation of the reproducibility coefficients were carried out considering the pair-wise ratings of all possible combinations of observers. For example, observer 1 and 2, observer 1 and 3, observer 1 and 4, and so on to observer 1 and 8 . Then observer 2 and 3, observer 2 and 4 , observer 2 and 5 and so on to observer 2 and 8 . This was done for each observer to the last observers 7 and 8 . For 8 observers and $30 \mathrm{X}$-rays this resulted in $28(8 \times 7 / 2)$ possible pairs times $30 \mathrm{X}$-rays, totalling 840 pairs of observations. Sample size and number of observers were chosen to accommodate the precision of the reproducibility estimates we wanted to achieve. When the number of observations is 840 , a 2 -sided $95 \%$ confidence interval (CI) for a reproducibility coefficient arbitrarily set at 0.40 will extend 0.05 around the point estimate (from 0.35 to 0.45 ). Statistical evaluations were carried out using SPSS 11.5 and StatXact 3.02 for Windows.

\section{Results}

Thirty patients with distal radial fractures were included. Of these patients, 15 fractures were of AO classification group A, 6 AO classification group B and 9 of AO classification group $\mathrm{C}$.

Table 1, shows the reproducibility coefficients for the direct quantitative X-ray measures and the template assessment technique. Slight and insignificant increases in ICCs (95\% CI) were observed for the radial length from 0.53 $(0.48 / 0.57)$ to $0.54(0.49 / 0.59)$ and volar angulation from $0.60(0.56 / 0.64)$ to $0.64(0.60 / 0.68)$. Marked improvements in reproducibility were observed for the radial inclination measurements. Intraclass correlations increased from 0.36 $(0.30 / 0.41)$ with the old technique to $0.49(0.43 / 0.55)$ with the template technique. The overall kappa for the Lidström score in the old technique was $0.37(0.31 / 0.43)$ and improved in the template technique to $0.59(0.52 / 0.63)$.

\section{Discussion}

Compared to the traditional quantitative technique, the template assessment technique resulted only in an improved inter-observer reproducibility for the radial inclination. There were slight improvements in reproducibility of the radial length and volar angulation. However, there was a notable improvement in the reproducibility of reduction results as assessed using the Lidström score.

The results of the quantative measurements of radial length and radial inclination are comparable to those reported by Kreder et al. They reported an inter-observer reliability coefficient of 0.49 for the radial length, and 0.32 for the AP radial angle [6]. As in our study, a better reproducibility was found for the volar angulation on the lateral view.

In this comparative study we had the same observers and the same patients with a 2-month interval and X-rays presented in another sequence order to overcome possible bias. The patients' sample was chosen to reflect the daily practice in which the AO type A fractures are more frequent than the Type B and C.

One could argue that the use of normal values for the traditional quantitative measurement technique would negatively affect its reproducibility since there is variation in

Table 1 Reproducibility coefficients for the X-ray measurements: old direct measurement technique versus template technique $(N=840$ observations)

\begin{tabular}{lll}
\hline Measure $^{\mathrm{a}}$ & $\begin{array}{l}\text { Old technique } \\
(95 \% \mathrm{CI})\end{array}$ & $\begin{array}{l}\text { Template technique } \\
(95 \% \mathrm{CI})\end{array}$ \\
\hline Radial length & $0.53(0.48 / 0.57)$ & $0.54(0.49 / 0.59)$ \\
Radial inclination & $0.36(0.30 / 0.41)$ & $0.49(0.43 / 0.55)$ \\
Volar angulation & $0.60(0.56 / 0.64)$ & $0.64(0.60 / 0.68)$ \\
Lidström & $0.37(0.31 / 0.43)$ & $0.59(0.52 / 0.63)$ \\
\hline
\end{tabular}

${ }^{a}$ Categorical data, weighted kappa, numerical data, ICC 
anatomical proportions between individuals in the population. For example, the ulnar length varies within $30 \%$ of the population [12]. Therefore, the use of normal values may lead to inaccurate classification of the reduction result. However, in our study, the comparison with the normal values did not negatively influenc the reproducibility, since all observers used the same values. By doing so, the only source of error left was the direct measurement of the fractured side. When the normal side is used to compare with in the classical measurement way, there would be measurement errors of the fractured side and of the uninjured side.

The improved reproducibility of the fractures deformity classification as expressed by the Lidström score (from 0.37 to 0.59 ) was primarily due to improvement in measurement of the radial inclination in the template technique. By using the template technique for the Lidström score, one could expect the most improvements in both the radial length and radial inclinations. The volar angulation would not be expected to improve much since it is measured independently from the other side (Fig. 1).

Surprisingly, we could not demonstrate an influence of the template technique for the radial length in this study, although it is partly dependent on the position of the radial axis on the PA view and could therefore benefit from templating. An explanation for this phenomenon could be that for measuring the difference in radial height (as shown in Fig. 3), there is still an axis needed. This becomes more eminent as the radial styloid is displaced more laterally (or rotated) and both tips of the radial styloid are not in line with the radial axis any more. Differences in placing the axis could be a source for variance, leading to a limited improvement in the Kappa value. In contrast, the radial inclination on the PA view in the template technique is basically independent of the radial axis and therefore more easy to measure with the template.

A limitation of the template technique is that it may not be applicable in patients with a bilateral fracture or patients with a history of a distal radial fracture or deformity on the other side. In this study, only patients with a unilateral distal radial fracture were included, reflecting the most common occurrence of distal radial fractures.

Another limitation of this study was that, in order to avoid learning curves, only experienced observers were used, which may not always be the case in daily practice. Also, the templates of the unaffected side were made by the same investigator. In daily practice, making the template could also be a source of variation since the correct position of the templates may be more difficult in case of severe dislocation or with slight differences in projection of the bones due to the angle of the X-ray beam. Perhaps the use of digital subtraction may be helpful to overcome this.

In conclusion, by using the template technique for $\mathrm{X}$-Ray measurement we improved the reproducibility of the classification of radiological dislocation of distal radius fractures which is a step to come to evidence based distal radial fracture treatment as outlined in two recent Cochrane reviews $[4,5]$. This procedure can also be performed for other radiological parameters such as ulnar variance. Future research should focus on the improvement of the template technique and the use of digital measurement-subtraction techniques/software modules in this time of filmless X-Ray pictures.

Acknowledgment We would like to thank the following raters for their time and willingness to participate in this study, A.H. vd Veen, trauma surgeon, Catharina ziekenhuis, Eindhoven; P. Kloen, Orthopeadic surgeon, Academic Medical Center Amsterdam; H.J.W. Koot, Orthopeadic surgeon, Máxima Medical Center Eindhoven, J.S.K. Luitse, trauma surgeon, Academic Medical Center, Amsterdam; D.R. Kool, trauma radiologist, Academisch Medisch Centrum, Amsterdam, C.N. van Dijk, professor of orthopaedic surgery Academic Medical Center, Amsterdam; M. van der Elst, trauma surgeon, Reinier de Graaf Ziekenhuis, Delft. There was no grant or funding received for this paper.

\section{References}

1. Lidstrom A (1959) Fractures of the distal end of the radius. A clinical and statistical study of end results. Acta Orthop Scand Suppl 41:1-118

2. Sarmiento A, Pratt GW, Berry NC, Sinclair WF (1975) Colles' fractures. Functional bracing in supination. J Bone Joint Surg Am $57: 311-317$

3. Handoll HH, Madhok R (2003) From evidence to best practice in the management of fractures of the distal radius in adults: working towards a research agenda. BMC Musculoskelet Disord 4:27

4. Handoll HH, Madhok R (2003) Surgical interventions for treating distal radial fractures in adults. Cochrane database. Syst Rev CD003209

5. Handoll HH, Madhok R (2003) Conservative interventions for treating distal radial fractures in adults. Cochrane database. Syst Rev CD000314

6. Kreder HJ, Hanel DP, McKee M, Jupiter J, McGillivary G, Swiontkowski MF (1996) X-ray film measurements for healed distal radius fractures. J Hand Surg Am 21:31-39

7. Goslings JC (1999) Flexafix: the development of a new dynamic external fixation device for the treatment of distal radial fractures (thesis/dissertation)

8. Oestern HJ (1999) Distal radius fractures. I. Basic principles and conservative therapy. Chirurg 70:1180-1192

9. Trumble TE, Culp RW, Hanel DP, Geissler WB, Berger RA (1999) Intra-articular fractures of the distal aspect of the radius. Instr Course Lect 48:465-480

10. Wiemer P, Koster G, Felderhoff J, Weber U (1999) [Fractures of the distal radius. Changing therapeutic strategies]. Orthopade 28:846-852

11. van der LW, Ericson R (1981) Colles' fracture. How should its displacement be measured and how should it be immobilized? J Bone Joint Surg Am 63:1285-1288

12. Freedman DM, Edwards GS Jr, Willems MJ, Meals RA (1998) Right versus left symmetry of ulnar variance. A radiographic assessment Clin Orthop Relat Res 354:153-158

13. Hollevoet N, Van Maele G, Van Seymortier P, Verdonk R (2000) Comparison of palmar tilt, radial inclination and ulnar variance in left and right wrists. J Hand Surg Br 25:431-433 
14. Schrout PE, Fleiss JL (1979) Intraclass Correlations: uses in assessing rater reliability. Psychol Bull 86:420-428

15. Fleiss JL, Cohen JT (1973) The equivalence of weighted kappa and the intraclass correlation coefficient as measures of reliability. Educ Psychol Meas 33:613-619
16. Landis JR, Koch GG (1977) The measurement of observer agreement for categorical data. Biometrics 33:159-7 